\title{
Characterization of Fricke-gelatin dosimeters for Intraoperative Radiation Therapy dosimetry
}

\author{
Gabriele Magugliani $^{2}{ }^{*}$, Giulia M. Liosi ${ }^{l}$, Stefano Andreoli ${ }^{2}$, Stefano De Crescenzo ${ }^{2}$, Eros Mossini ${ }^{l}$, Elena Macerata ${ }^{l}$ \\ and Mario Mariani ${ }^{1}$ \\ *gabriele.magugliani@polimi.it \\ ${ }^{1}$ Department of Energy, Nuclear Engineering Division, Politecnico di Milano, Milano, Italy \\ ${ }^{2}$ UOC Fisica sanitaria, ASST Papa Giovanni XXIII, Bergamo, Italy
}

\begin{abstract}
$\underline{\text { Abstract }}$
In this work, the usability of Fricke gels in Intraoperative Radiation Therapy (IORT) dosimetry was investigated. Irradiated gel systems are characterized by means of UV/Vis spectrophotometric analysis in order to define the main dosimetric indexes of interest and to evaluate their postirradiation stability. A measurement of Output Factors for different field sizes is also attempted to study the application of this system for routine quality control. Ad-hoc manufactured thin-layers of gel are employed to perform acquisitions of planar transverse dose profiles and to quantify the effect of Fe(III) diffusion. The Fricke gel system presents an adequate dosimetric performance, with excellent response linearity up to $30 \mathrm{~Gy}$. Comparison of dosimetric response over a wide range of irradiation dose rates allows to conclude that the gel response is not influenced significantly by this parameter. Transverse dose profiles acquired with thin-layers present an agreement within 3\% with reference values, even in very steep dose gradient regions. Finally, computed values for the diffusion coefficient of ferric ions are in good agreement with those reported in literature for dosimetric gels of similar composition. Overall performance of the gel system appears promising for further applications in IORT dosimetry.
\end{abstract}

Keywords: IORT, dosimetry, Fricke gel, output factor, 2D dose profiling, diffusion coefficient.

\section{Introduction}

IntraOperative Radiation Therapy (IORT) is a special kind of radiotherapy that is based on the delivery of therapeutic dose during surgical intervention, typically after resection of the tumoral mass (Gunderson et al., 1999). The treatment beam may be provided by conventional external beam radiotherapy (EBRT) LINACs, but their use to this purpose is not practical, since mid-surgery transport of the patient to an irradiation bunker adds non negligible risks and complexity the procedure. To overcome this problem, dedicated IORT accelerators have been developed in recent years: these machines can be installed directly in the operating room, thus eliminating said logistical problems.

The Novac11 (SIT, Italy) is an example of dedicated mobile LINAC which is used for electronbeam IORT. The Novac11 can produce an electron beam at four pre-set nominal energies of 4, 6,8 , and $10 \mathrm{MeV}$, which is then collimated by a hard docking applicator used to focus and guide the radiation field to the target site. Different cylindrical applicators, ranging from $100 \mathrm{~mm}$ down to 30 $\mathrm{mm}$ in diameter, are available in order to satisfy specific clinical requirements. Novac11 shares 
many technical features with similar accelerators of the same model family such as the Novac7 (Pimpinella et al., 2007): in particular, the produced electron beam is characterized by a very high value of dose-per-pulse, which can reach values in the range of $10 \mathrm{cGy}$ per pulse depending on the acceleration energy and the applicator in use.

Generally, international codes of practice recommend the use of parallel plate ionization chambers for dose measurement in high-energy electron fields (IAEA TRS 398, 2000). Under very high values of dose-per-pulse however, correction factors for ion recombination $\left(k_{s}\right)$ calculated according to such codes of practice are not adequate, since they can lead to significant errors in dose evaluations (Di Martino et al., 2005; Piermattei et al., 2000). This effect is due to the high fraction of free electrons present in the camber volume, which impacts the collection efficiency at values of dose-per-pulse > $10 \mathrm{mGy}$ per pulse (Laitano et al., 2006). Several models have been developed to face the problem of determining adequate $k_{s}$ values in high dose-per-pulse fields (Di Martino et al., 2005; Laitano et al., 2006; Piermattei et al., 2000), thus allowing the use of ionization chambers with this particular kind of beams. Another approach to perform dosimetric measurements at such high values of dose-per-pulse consists in employing dosimetric systems which present a response independent from irradiation dose rate; in this way, the need for saturation corrections can be eliminated altogether. This is the case, for example, of Fricke standard dosimeters and alanine dosimeters: thanks to their dose rate independence (McEwen et al., 2015; Rogers, 1987), they are one of the instruments adopted for absolute reference dosimetry in IORT (Rosi and Viti, 2003). Their use however is very laborious when compared to ionization chambers, often requiring the support of a metrology center for their readout.

In this work the usability of Fricke gels in IORT dosimetry, both under reference and non-reference conditions, was investigated in order to identify possible advantages over more conventional dosimetric systems. The basic components of Fricke gels consist in a gel matrix, Fe(II) ions (typically in the form of Ferrous Ammonium Sulfate, FAS), and the selective ligand Xylenol Orange (XO) (Schreiner, 2004). Radiolytic products resulting mainly from water radiolysis oxidize quantitatively $\mathrm{Fe}$ (II) ions present in solution in a dose-dependent manner. Resulting $\mathrm{Fe}(\mathrm{III})$ ions are then chelated by the selective ligand $\mathrm{XO}$, leading to the formation of a low mobility XO-Fe(III) complex. This complex exhibits an intense optical absorption peak centered at $585 \mathrm{~nm}$ whose intensity is linearly related to absorbed dose. The intrinsic tridimensional nature of Fricke gels, which allows to manufacture them in arbitrary shapes (Schreiner, 2004), coupled with their ability to perform direct spatial dose mapping in inhomogeneous phantoms (Gum et al., 2002), are the key features that lead the interest for the research in this field.

In order to exploit the mentioned capabilities of Fricke gels also in the field of IORT, it is however necessary to verify whether this gel system presents an adequate dosimetric performance under such peculiar irradiation fields. While some data exists in literature about the characterization of Fricke gels' dose-response with different matrices under various dose rates (Gallo et al., 2019; Olsson et al., 1989; Schulz et al., 1990; Soliman et al., 2017), no studies have been carried out with the very high values of dose-per-pulse characteristic of Novac11, to the best of the authors' knowledge. This preliminary dose-response characterization was therefore an integral part of this study, which was then followed by more specialized dosimetric investigations regarding planar dose profiling and evaluation of stability properties of the gel system. This last step is of particular relevance since physical stability is the main factor that influences the integrity of acquired planar and volumetric 
dose distributions, and in particular an evaluation of the diffusion coefficient of the XO-Fe(III) complex can help quantify the post-irradiation spatial stability of the system (Schreiner, 2004).

\section{Materials and Methods}

In order to evaluate the applicability of Fricke gels to IORT dosimetry, the logical steps here reported were followed:

1 characterization of dosimetric performances of Fricke gels irradiated with very high doseper-pulse electron beams generated by a Novac11 under reference conditions - comparison of Fricke gel response with irradiations performed by a conventional linear accelerator (Clinac ${ }^{\circ}$ iX DHX, Varian Medical Systems Inc., USA);

2 evaluation of Fricke gel applicability for the measurement of Output Factors (OF) for different Novac11 applicator sizes;

3 acquisition of Novac11 2D transverse dose distributions and comparison with reference data;

4 quantification of the diffusion coefficient of XO-Fe(III) complex.

\subsection{Samples preparation}

Fricke gel dosimeters prepared for this study consisted in a solution of $0.125 \mathrm{mM} \mathrm{XO}$ disodium salt, $0.5 \mathrm{mM}$ FAS, $25 \mathrm{mM} \mathrm{H}_{2} \mathrm{SO}_{4}$ and $3 \mathrm{~g}$ of gelatin (300 bloom, type A, from porcine skin) per $100 \mathrm{ml}$ of final product.

To prepare the dosimetric solution, gelatin was dissolved in half the final water volume by firstly allowing it to swell under stirring for 5 minutes, and then by heating up to $50{ }^{\circ} \mathrm{C}$. Once complete dissolution was achieved, the solution was allowed to cool down to $35{ }^{\circ} \mathrm{C}$. The remaining $50 \%$ of the final water volume was acidified with sulfuric acid and used to prepare two separate $\mathrm{XO}$ and FAS solutions of equal volume. The XO solution was added to the gel, followed by the FAS solution. Some time was allowed to achieve homogeneity under stirring. The dosimetric solution was then poured in the appropriate PMMA containers and kept in the dark at $6-8^{\circ} \mathrm{C}$ for at least $12 \mathrm{~h}$ to allow complete gelation. Both spectrophotometer cuvettes $\left(1-\mathrm{x}-1-\mathrm{x}-4.5 \mathrm{~cm}^{3}\right)$ and thin-layers (Gambarini et al., 2011; Tomatis et al., 2007) samples (180-x-180-x-2 mm ${ }^{3}$ ) were prepared. Gel containers were sealed with Parafilm to prevent gel drying and oxygen ingress. Multiple gel batches were manufactured in order to evaluate the reproducibility of the dosimetric system. Prior to irradiation and optical analysis, samples were allowed to thermalize to room temperature $\left(25^{\circ} \mathrm{C}\right)$ (Davies and Baldock, 2010).

\subsection{Irradiation}

Cuvette samples were employed to characterize the dosimetric performance and to evaluate the OFs for different Novac11 applicator sizes. For this purpose, cuvettes samples underwent a uniform irradiation. Thin-layer samples were exploited to obtain Novac11 transverse dose profiles and to evaluate the diffusion process.

Regarding the Novac11 IORT accelerator (installed at ASST Papa Giovanni XXIII, Bergamo, Italy), the 8 and $10 \mathrm{MeV}$ nominal acceleration energies were employed in this study. A liquid water phantom was used for irradiations. Samples were positioned with their reference measurement 
point, defined as the geometrical center of the specimen, at the depth of maximum dose along the beam axis (14 and $15 \mathrm{~mm}$ for 8 and $10 \mathrm{MeV}$, respectively). This geometric configuration guaranteed a dose uniformity $>99 \%$ over the volume of cuvette samples. As a preliminary step, Novac11 was calibrated in terms of $\mathrm{Gy} \mathrm{MU}^{-1}$ with the use of an Advanced Markus ionization chamber (34045, PTW) according to IAEA TRS-398 protocol and by adopting the method proposed by Laitano et al. (Laitano et al., 2006) to determine the ion recombination correction factor $k_{s}$.

The $100 \mathrm{~mm}$ reference applicator was used for characterization of the gel response. For this purpose, cuvette samples were uniformly irradiated in the range $0-50 \mathrm{~Gy}$, for both 10 and $8 \mathrm{MeV}$ energies. To draw a comparison with more conventional irradiation conditions, a Clinac $₫$ iX DHX (Varian Medical Systems Inc., installed at Humanitas Gavazzeni, Bergamo, Italy) was also employed. In this case, samples were irradiated over the same dose range at $6 \mathrm{MV}-600 \mathrm{MU} \mathrm{min}^{-1}$ by positioning in a RW3 slab phantom (dose uniformity $>99 \%$ over the sensitive volume of the gel).

Subsequently, for the OF evaluation, $80 \mathrm{~mm}, 70 \mathrm{~mm}$ and $60 \mathrm{~mm}$ Novac11 applicators were used. Output factor for applicator size $x$ is defined as (IAEA TRS 398, 2000):

$$
\left.O F\right|^{x}=\frac{\left.\frac{D}{M U}\right|^{x}}{\left.\frac{D}{M U}\right|^{100}}
$$

where the numerator and the denominator represent the dose per Monitor Unit (MU) delivered at the reference measurement point by using the applicator of diameter $x$ and by using the $100 \mathrm{~mm}$ reference applicator, respectively. For this purpose, multiple cuvettes samples were uniformly irradiated with the same value of MU (191 and $228 \mathrm{MU}$ for acceleration energies 10 and $8 \mathrm{MeV}$, respectively), and the ratio between the measured doses for applicator $x$ and for the reference applicator was computed. This step was performed after the dosimetric characterization of the gel in order to guarantee that the selected values of MU corresponded to dose values that laid in the linear response region of the gels.

Thin-layers were used for the evaluation of transverse dose profiles. For this purpose, samples were positioned at buildup depth orthogonally to the beam axis and irradiated at $20 \mathrm{~Gy}$ (100 mm applicator $-10 \mathrm{MeV}$ ).

\subsection{Optical analysis}

Optical analysis of cuvette samples was performed with a LAMBDA 650 UV-Vis spectrophotometer (PerkinElmer, Inc.) by evaluating the intensity of the XO-Fe(III) absorption peak at $585 \mathrm{~nm}$. Net absorbance values $(\triangle A b s)$ for irradiated samples were obtained by subtracting the absorbance of blank non-irradiated samples. Planar thin-layers were analyzed with a commercial reflection scanner (300 DPI resolution) in order to acquire bidimensional RGB images of the optical density, which is linearly related to absorbed dose. Optical analyses of cuvette samples were performed starting from $12 \mathrm{~h}$ after irradiation and repeated up to several days after irradiation in order to evaluate the chemical stability of the system. This timing was optimized in order to obtain a standardized and repeatable protocol. 
Characterization of the performance of the gels allowed to quantify the dosimetric parameters of interest: dose sensitivity, dose resolution and post-irradiation stability. The dose sensitivity $S$ was defined as the slope of the linear fit in the linear dose range of response in the $\triangle A b s$-D plot. Dose resolution was calculated as reported elsewhere (Magugliani et al., 2018). Throughout this work, all reported confidence intervals represent $1 \sigma$ of uncertainty.

Finally, the diffusion coefficient of XO-Fe(III) was determined. This is a key parameter that can quantify the post-irradiation spatial stability of acquired dose distributions. Even if the diffusion phenomenon in Fricke gels has already been extensively characterized, this work aimed at confirming literature data under the harsh IORT irradiation conditions. To perform this task, repeated optical analysis of a selected sample at increasing time intervals after irradiation (from $2 \mathrm{~h}$ up to $24 \mathrm{~h}$ ) were performed. A finite element method (FEM) approach, similar to some already proposed in literature (Harris et al., 1996; Vedelago et al., 2014), was used to numerically solve the diffusion equation and to determine the value of the diffusion coefficient. In particular, a FEM implemented in the MATLAB environment was applied to the bidimensional dose distribution previously acquired with thin-layers used for transverse dose profiling.

\section{Results}

\subsection{Dosimetric characterization}

Dosimetric parameters obtained from the analysis of uniformly irradiated cuvette samples $12 \mathrm{~h}$ after irradiation are reported in Table 1 . An excellent dose response linearity $\left(R^{2}>0.995\right)$ was obtained for all irradiation conditions in the range 5 - $30 \mathrm{~Gy}$. Reproducibility between different gel batches was within $3 \%$. By way of example, the linear $\triangle A b s$-D relationship for Novac11 irradiation with $10 \mathrm{MeV}$ nominal energy is reported in Figure 1.

Deviations from linearity were evident for doses lower than $2.5 \mathrm{~Gy}$, therefore, for the definition of dosimetric parameters only doses in the range 5-30 Gy were considered. As reported in literature, this drawback could be eliminated by subjecting all samples to a uniform pre-irradiation of ca. $5 \mathrm{~Gy}$ before their actual use in the field (Liosi et al., 2018). This approach however should be undertaken with care, as it could be source of additional uncertainties.

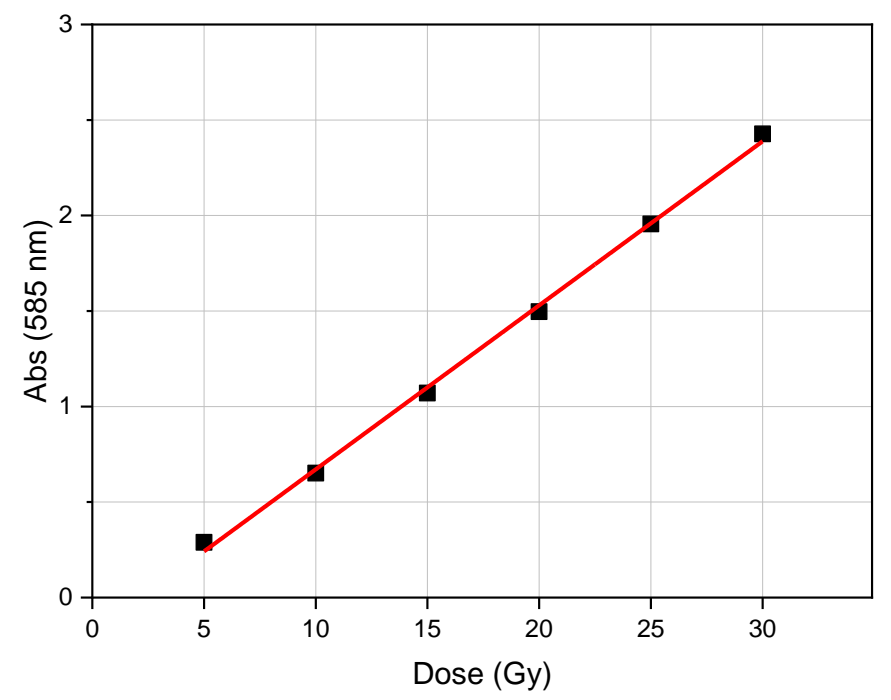


Table 1. Summary of dosimetric parameters of Fricke gels with respect to different irradiation methods (analysis $12 \mathrm{~h}$ post-irradiation). Irradiation with NOVA C11 performed under reference conditions (100 mm applicator).

\begin{tabular}{|c|c|c|c|}
\hline & Novac11, $10 \mathrm{MeV}$ & Novac11, $8 \mathrm{MeV}$ & Clinac ${ }^{-}$ \\
\hline Sensitivity [Gy $\left.{ }^{-1}\right]$ & $0.087 \pm 0.001$ & $0.087 \pm 0.005$ & $0.087 \pm 0.001$ \\
\hline Avg. dose resolution [Gy] & $0.34 \pm 0.05$ & $0.20 \pm 0.02$ & $0.24 \pm 0.02$ \\
\hline Avg. accuracy & \multicolumn{3}{|c|}{$\approx 2 \%$} \\
\hline Avg. precision & \multicolumn{3}{|c|}{$<\%$} \\
\hline
\end{tabular}

Regarding stability of the gel response, the best dosimetric performances were obtained $12 \mathrm{~h}$ post-irradiation. Nevertheless, a good temporal stability was observed over all gel batches, with variations in sensitivity on the order of $1 \%$ per day. Deterioration of dose resolution was more significant after $24 \mathrm{~h}$ from irradiation for all gel batches and irradiation types due to worsening of sample precision.

From the collected data for samples irradiated with Novac11 and Clinac® LINAC, no significant differences, nor in terms of dosimetric performance, nor in terms of post-irradiation stability, were found despite the profound differences in beam characteristics.

\section{$\underline{3.2 \mathrm{OF} \text { measurement }}$}

OF values measured with Fricke gels are reported in Table 2 . Reference values ${ }^{1}$ were determined during accelerator commissioning with an Advanced Markus ionization chamber (34045, PTW) following international dosimetry protocols (IAEA TRS 398, 2000) and by adopting the method proposed by Laitano et al. (Laitano et al., 2006) to determine the ion recombination correction factor $k_{s}$. A good agreement within $2 \%$ for all combinations of investigated irradiation energies and applicator diameters can be noted.

Table 2. Comparison between reference OF and values measured with Fricke gels for the Novac11.

\begin{tabular}{|c|c|c|c|c|c|c|}
\hline \multirow{2}{*}{ Applicator } & \multicolumn{3}{|c|}{ OF - 8 MeV } & \multicolumn{3}{c|}{ OF - 10 MeV } \\
\cline { 2 - 7 } & Fricke gel & Reference & \% difference & Fricke gel & Reference & $\%$ difference \\
\hline $80 \mathrm{~mm}$ & $1.55 \pm 0.02$ & 1.556 & $-0.51 \%$ & $1.54 \pm 0.01$ & 1.549 & $-0.45 \%$ \\
\hline $70 \mathrm{~mm}$ & $1.68 \pm 0.01$ & 1.685 & $-0.50 \%$ & $1.70 \pm 0.01$ & 1.671 & $+1.80 \%$ \\
\hline $60 \mathrm{~mm}$ & $1.85 \pm 0.01$ & 1.844 & $+0.10 \%$ & $1.84 \pm 0.05$ & 1.824 & $+1.10 \%$ \\
\hline
\end{tabular}

\subsection{Transverse dose profile}

In a preliminary investigation, not reported in this work for sake of brevity, a linear relation between PV (Pixel Value of each separate RGB channel of acquired images of irradiated thinlayers) and absorbed dose was confirmed. In particular $\mathrm{PV}_{\mathrm{G}}$, i.e. the pixel value of the Green RGB channel, showed a superior linearity $\left(\mathrm{R}^{2}>0.99\right)$, extending up to $25 \mathrm{~Gy}$, allowing for better accuracy and precision in resulting dose evaluation over the entire considered dose range. Therefore, after such calibration, grayscale $\mathrm{PV}_{\mathrm{G}}$ images were converted pixel-by-pixel to dose images.

\footnotetext{
1 As suggested in literature (Di Venanzio et al., 2015; Pimpinella et al., 2019) for similar experimental setups, an uncertainty of $1.5 \%$ in reference $\mathrm{OF}$ values can be assumed. This value can be derived by combining uncertainties in the ratio between $k_{s}$ values for different applicator sizes $(\approx 1 \%)$, accelerator output $(<1 \%)$ and reproducibility of chamber signal $(0.2 \%)$.
} 
For dose profiling, thin-layers were irradiated to $20 \mathrm{~Gy}$ with the $100 \mathrm{~mm}$ applicator (10 $\mathrm{MeV}$ energy). For example, Figure 2 illustrates a transverse dose profile extracted along the diametral direction, superimposed to reference dose values measured with a microDiamond detector (60019, PTW). Thanks to the high DPI number of acquired images, resulting in a very fine pixel subdivision, the resulting 2D dose profiles can be considered macroscopically quasi-continuous. In order to increase SNR, averages between adjacent pixels were considered, resulting in a final inplane spatial resolution of $0.5 \times 0.5 \mathrm{~mm}^{2}$.

All data points of the measured dose distribution were in agreement with the $3 \% / 3 \mathrm{~mm}$ criterion (De Deene and Vandecasteele, 2013) when compared to the reference microDiamond dose profile. No significant differences were noted between multiple thin-layers irradiated under the same conditions, thus supporting the reproducibility of this kind of measurement. It must be noted that for relative dose values $<10 \%$, Fricke gels systematically underestimates reference dose values; this fact, albeit undesirable, is coherent to what was noted during calibration with cuvettes.

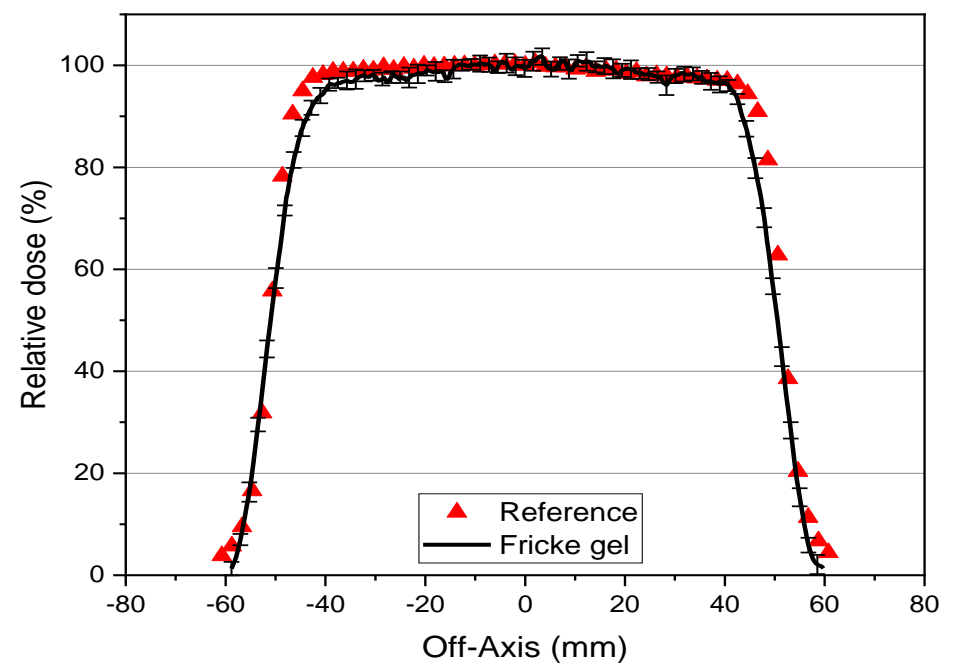

Figure 2. Transverse dose profile measured with Fricke gel thin-layer (10 MeV energy, $100 \mathrm{~mm}$ applicator) expressed as a function of lateral deviation from beam axis. Triangles represent reference values measured with microDiamond detector (60019, PTW).

\subsection{Diffusion evaluation}

The evaluation of the diffusion coefficient was performed starting from the $2 \mathrm{D}$ dose profiles acquired with thin-layers, measured from $2 \mathrm{~h}$ up to $24 \mathrm{~h}$ after irradiation at different time intervals. A FEM approach was used to solve the diffusion equation and estimate the diffusion coefficient.

Diffusion of XO-Fe(III) complex inside the thin-layer can be described by the general 2D diffusion equation, coupled with no flux at boundaries condition:

$$
\frac{\partial C}{\partial t}=\alpha \nabla^{2} C
$$

In detail, $\alpha$ is the diffusion coefficient to be determined and $C=C(x, y, t)$ is the local concentration of $\mathrm{XO}-\mathrm{Fe}(\mathrm{III})$ complex at position $x, y$ at time $t$. As already mentioned, $C$ is linearly related to the dose, and therefore to the measured value of $\mathrm{PV}_{\mathrm{G}}$ in irradiated thin-layers. 
The logical workflow employed for the determination of the diffusion coefficient is depicted in Figure 3 and can be schematized as follows (the explicit dependence of $C$ on $x, y$ will not be indicated for simplicity):

- the initial spatial concentration $C_{e x}\left(t_{0}\right)$ is extrapolated by scanning an irradiated thin layer at post-irradiation time $t=t_{0} . C_{e x}\left(t_{0}\right)$ will be used as the initial condition by the solver;

- the geometry is discretized by constructing a mesh and a solution $C_{c}\left(t_{f}\right)$ is computed at a post-irradiation time $t_{f}>t_{0}$ by using a trial value of the diffusion coefficient $\alpha$;

- $C_{c}\left(t_{f}\right)$ is compared with the corresponding profile $C_{e x}\left(t_{f}\right)$ acquired by the same thin-layer previously employed to define $C_{e x}\left(t_{0}\right) . C_{e x}\left(t_{f}\right)$ will be the target function for the solver;

- a residual sum of squares (RSS) bidimensional profile is computed via a point-by-point comparison of $C_{c}\left(t_{f}\right)$ and $C_{e x}\left(t_{f}\right)$. The optimal value of $\alpha$ is achieved via an iterative process that minimizes the RSS.

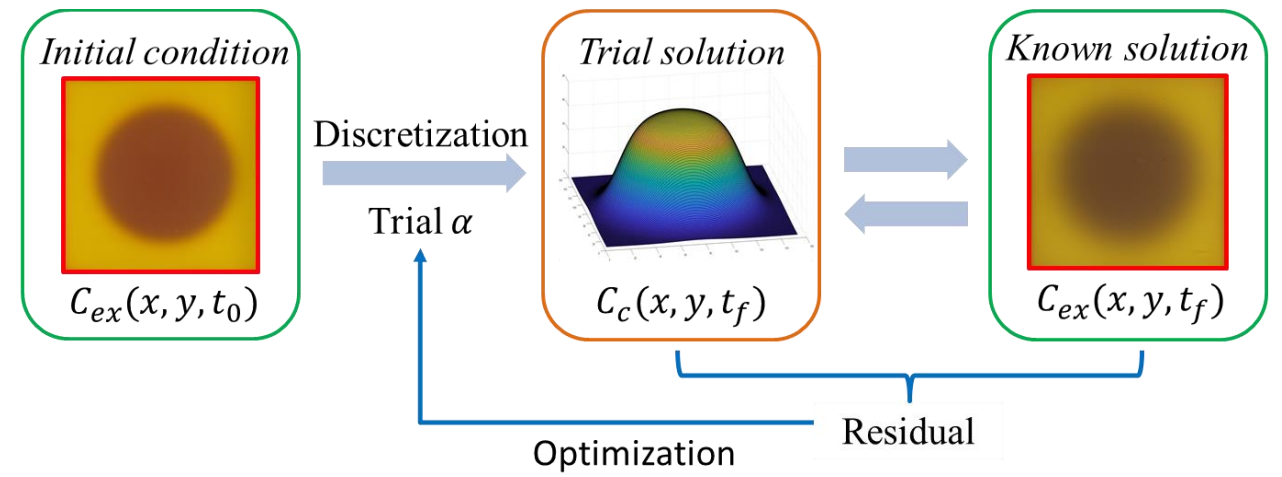

Figure 3. Workflow employed for the determination of the diffusion coefficient via FEM.

After averaging on multiple samples, the computed value for the diffusion coefficient is $0.81 \pm 0.05 \mathrm{~mm}^{2} \mathrm{~h}^{-1}$. The diffusion coefficient calculated in this work is in good agreement with those reported in literature for gels of similar composition (Baldock et al., 2001).

\section{Conclusions}

Fricke gels proved to be adequate instruments to perform dosimetry in IORT. Comparison between Novac11 and Clinac ${ }^{\circledR}$ irradiations strongly suggests that the response of the gel system is not influenced by the very high dose-per-pulse characteristic of such dedicated IORT LINAC, nor by the irradiation energy in the investigated range (6 to $10 \mathrm{MeV})$. Such independence of the dose response from beam characteristics is a desirable feature for any dosimetric system, since it limits dose evaluation uncertainties in applications where beam quality and dose rate are not known $a$ priori.

Moreover, Fricke gels presented an accuracy and precision in the evaluation of OF which is on par with the performance of other dosimetric systems employed in similar high dose-per-pulse irradiation conditions (Pimpinella et al., 2019). Ease in handling and analysis of Fricke gels are factors that could promote the employment of this system for routine quality control. Moreover, thanks to their accuracy and precision, they could also be exploited for intercomparison with conventional dosimetric systems such as ionization chambers. 
The intrinsic tridimensional response of gel dosimeters is very appealing for more advanced dosimetric tasks. In this work we have shown that these systems are fit for planar dose imaging; the results obtained are promising for the future development of this dosimetric application, as well as for a further extension to tridimensional dose measurements. In this perspective, having a reliable estimation of the diffusion coefficient value and of its reproducibility between different gel batches is fundamental for the definition of a protocol of use, since the diffusion phenomenon reduces the available time to acquire a reliable dose map distribution.

\section{$\underline{\text { Acknowledgement }}$}

Authors would like to thank the Radiodiagnostic Department of Humanitas Research Hospital Gavazzeni (Bergamo, Italy) for the access to LINAC irradiation facility.

\section{$\underline{\text { References }}$}

Baldock, C., Harris, P.J., Piercy, A.R., Healy, B., 2001. Experimental determination of the diffusion coefficient in twodimensions in ferrous sulphate gels using the finite element method. Australas. Phys. Eng. Sci. Med. https://doi.org/10.1007/BF03178282

Davies, J.B., Baldock, C., 2010. Temperature dependence on the dose response of the Fricke-gelatin-xylenol orange gel dosimeter. Radiat. Phys. Chem. https://doi.org/10.1016/j.radphyschem.2009.11.014

De Deene, Y., Vandecasteele, J., 2013. On the reliability of 3D gel dosimetry, in: Journal of Physics: Conference Series. https://doi.org/10.1088/1742-6596/444/1/012015

Di Martino, F., Giannelli, M., Traino, A.C., Lazzeri, M., 2005. Ion recombination correction for very high dose-perpulse high-energy electron beams. Med. Phys. https://doi.org/10.1118/1.1940167

Di Venanzio, C., Marinelli, M., Tonnetti, A., Verona-Rinati, G., Falco, M.D., Pimpinella, M., Ciccotelli, A., De Stefano, S., Felici, G., Marangoni, F., 2015. Characterization of a microDiamond detector in high-dose-per-pulse electron beams for intra operative radiation therapy. Phys. Medica. https://doi.org/10.1016/j.ejmp.2015.06.008

Gallo, S., Artuso, E., Brambilla, M.G., Gambarini, G., Lenardi, C., Monti, A.F., Torresin, A., Pignoli, E., Veronese, I., 2019. Characterization of radiochromic poly(vinyl-alcohol)-glutaraldehyde Fricke gels for dosimetry in external X-ray radiation therapy. J. Phys. D. Appl. Phys. https://doi.org/10.1088/1361-6463/ab08d0

Gambarini, G., Carrara, M., Rrushi, B., Guilizzoni, R., Borroni, M., Tomatis, S., Pirola, L., Battistoni, G., 2011. Characterization of Fricke-gel layers for absolute dose measurements in radiotherapy, in: ANIMMA 2011 Proceedings: 2nd International Conference on Advancements in Nuclear Instrumentation, Measurement Methods and Their Applications. https://doi.org/10.1109/ANIMMA.2011.6172969

Gum, F., Scherer, J., Bogner, L., Solleder, M., Rhein, B., Bock, M., 2002. Preliminary study on the use of an inhomogenous anthropomorphic Fricke gel phantom and 3D magnetic resonance dosimetry for verification of IMRT treatment plans. Phys. Med. Biol. https://doi.org/10.1088/0031-9155/47/7/401

Gunderson, L.L., Calvo, F.A., Willett, C.G., Harrison, L.B., Santos, M., 1999. General Rationale and Historical Perspective of Intraoperative Irradiation, in: Intraoperative Irradiation. https://doi.org/10.1007/978-1-59259-6966_1

Harris, P.J., Piercy, A., Baldock, C., 1996. A method for determining the diffusion coefficient in Fe(II/III) radiation dosimetry gels using finite elements. Phys. Med. Biol. https://doi.org/10.1088/0031-9155/41/9/013

IAEA TRS 398, 2000. Absorbed Dose Determination in External Beam Radiotherapy. At. Energy. https://doi.org/10.1097/00004032-200111000-00017

Laitano, R.F., Guerra, A.S., Pimpinella, M., Caporali, C., Petrucci, A., 2006. Charge collection efficiency in ionization chambers exposed to electron beams with high dose per pulse. Phys. Med. Biol. https://doi.org/10.1088/0031$9155 / 51 / 24 / 009$ 
Liosi, G.M., Gambarini, G., Artuso, E., Benedini, S., Macerata, E., Giacobbo, F., Gargano, M., Ludwig, N., Carrara, M., Pignoli, E., Mariani, M., 2018. Study on Characteristics of Fricke Xylenol Orange Gelatin Dosimeters. IEEE Trans. Nucl. Sci. https://doi.org/10.1109/TNS.2018.2858145

Magugliani, G., Liosi, G.M., Tagliabue, D., Mossini, E., Negrin, M., Mariani, M., 2018. Characterization of PAGAT dose response upon different irradiation conditions. Radiat. Eff. Defects Solids. https://doi.org/10.1080/10420150.2018.1528604

McEwen, M., Sharpe, P., Vörös, S., 2015. Evaluation of alanine as a reference dosimeter for therapy level dose comparisons in megavoltage electron beams. Metrologia. https://doi.org/10.1088/0026-1394/52/2/272

Olsson, L.E., Petersson, S., Ahlgren, L., Mattsson, S., 1989. Ferrous sulphate gels for determination of absorbed dose distributions using MRI technique: Basic studies. Phys. Med. Biol. https://doi.org/10.1088/0031-9155/34/1/004

Piermattei, A., Delle Canne, S., Azario, L., Russo, A., Fidanzio, A., Miceli, R., Soriani, A., Orvieto, A., Fantini, M., 2000. The saturation loss for plane parallel ionization chambers at high dose per pulse values. Phys. Med. Biol. https://doi.org/10.1088/0031-9155/45/7/312

Pimpinella, M., Andreoli, S., De Angelis, C., Della Monaca, S., D’Arienzo, M., Menegotti, L., 2019. Output factor measurement in high dose-per-pulse IORT electron beams. Phys. Medica. https://doi.org/10.1016/j.ejmp.2019.04.021

Pimpinella, M., Mihailescu, D., Guerra, A.S., Laitano, R.F., 2007. Dosimetric characteristics of electron beams produced by a mobile accelerator for IORT. Phys. Med. Biol. https://doi.org/10.1088/0031-9155/52/20/008

Rogers, D.W.O., 1987. Introduction to Radiological Physics and Radiation Dosimetry by F. H. Attix . Med. Phys. https://doi.org/10.1118/1.596041

Rosi, A., Viti, V., 2003. Guidelines for quality assurance in intra-operative radiation therapy. ISTISAN Report 03/1 EN, Istituto Superiore di Sanità.

Schreiner, L.J., 2004. Review of Fricke gel dosimeters. J. Phys. Conf. Ser. https://doi.org/10.1088/1742-6596/3/1/003

Schulz, R.J., DeGuzman, A.F., Nguyen, D.B., Gore, J.C., 1990. Dose-response curves for Fricke-infused agarose gels as obtained by nuclear magnetic resonance. Phys. Med. Biol. https://doi.org/10.1088/0031-9155/35/12/002

Soliman, Y.S., El Gohary, M.I., Abdel Gawad, M.H., Amin, E.A., Desouky, O.S., 2017. Fricke gel dosimeter as a tool in quality assurance of the radiotherapy treatment plans. Appl. Radiat. Isot. https://doi.org/10.1016/j.apradiso.2016.12.004

Tomatis, S., Carrara, M., Gambarini, G., Marchesini, R., Valente, M., 2007. Gel-layer dosimetry for dose verification in intensity-modulated radiation therapy. Nucl. Instruments Methods Phys. Res. Sect. A Accel. Spectrometers, Detect. Assoc. Equip. https://doi.org/10.1016/j.nima.2007.05.216

Vedelago, J., Quiroga, A., Valente, M., 2014. Characterization of ferric ions diffusion in Fricke gel dosimeters by using inverse problem techniques. Radiat. Eff. Defects Solids. https://doi.org/10.1080/10420150.2014.958749 